\title{
Modified theoretical minimum emittance lattice for an electron storage ring with extreme-low emittance
}

\author{
Yi Jiao, ${ }^{*}$ Yunhai Cai, and Alexander Wu Chao \\ SLAC National Accelerator Laboratory, Menlo Park, California 94025, USA
}

(Received 25 January 2011; published 5 May 2011)

\begin{abstract}
In the continuing efforts to reduce the beam emittance of an electron storage ring composed of theoretical minimum emittance (TME) lattice, down to a level of several tens of picometers, nonlinear dynamics grows to be a great challenge to the performance of the storage ring because of the strong sextupoles needed to compensate for its large global natural chomaticities coupled with its small average dispersion function. To help in dealing with the challenge of nonlinear optimization, we propose a novel variation of theoretical minimum emittance (TME) lattice, named as "modified-TME" lattice, with minimal emittance about 3 times of the exact theoretical minimum, while with more compact layout, lower phase advance per cell, smaller natural chromaticities, and more relaxed optical functions than that in a TME cell, by using horizontally defocusing quadrupole closer to the dipole or simply combinedfunction dipole with horizontally defocusing gradient. We present approximate scaling formulas to describe the relationships of the design parameters in a modified-TME cell. The applications of modified-TME lattice in the PEP-X storage ring design are illustrated and the proposed lattice appears a good candidate for synchrotron radiation light source with extremely low emittance.
\end{abstract}

DOI: 10.1103/PhysRevSTAB.14.054002

PACS numbers: 29.20.db, 41.85.-p, 29.27.Bd

\section{INTRODUCTION}

Emittance is one of the most important parameters for electron storage rings. Extremely low emittance allows high luminosity in colliders or high brightness in synchrotron light sources. During the past few decades, many efforts have been made to study the theoretical minimum emittance (TME) [1,2] and its concrete implementations (e.g., $[3,4])$.

Because of the equilibrium between the radiation damping and quantum fluctuation, the horizontal natural emittance $\varepsilon_{x}$ of electron beam in a storage ring is given by [5]

$$
\varepsilon_{x}=C_{q} \gamma_{L}^{2} \frac{\langle H\rangle_{\text {dipole }}}{J_{x} \rho}
$$

where $C_{q}=3.83 \times 10^{-13} \mathrm{~m} ; \gamma_{L}$ is the Lorenz factor; $J_{x}$ is the horizontal damping partition number; $\rho=L_{B} / \theta$ is the bending radius of dipole, with $L_{B}$ the dipole length and $\theta$ the bending angle; and $\langle H\rangle$ is averaged over the storage ring dipoles of the function

$$
H=\gamma_{x} D_{x}^{2}+2 \alpha_{x} D_{x} D_{x}^{\prime}+\beta_{x} D_{x}^{\prime 2},
$$

where $\alpha_{x}, \beta_{x}$, and $\gamma_{x} \equiv\left(1+\alpha_{x}^{2}\right) / \beta_{x}$ are the CourantSnyder parameters [6], $D_{x}$ and $D^{\prime}{ }_{x}$ are the dispersion and its derivative, respectively. For simplicity, the subscript $x$

\footnotetext{
*jiaoyi@slac.stanford.edu
}

Published by the American Physical Society under the terms of the Creative Commons Attribution 3.0 License. Further distribution of this work must maintain attribution to the author(s) and the published article's title, journal citation, and DOI. of the optical functions will be dropped in the following text.

In a storage ring with uniform dipoles, $J_{x} \approx 1$, the TME is derived by minimizing $\langle H\rangle_{\text {dipole }}$ with symmetric dispersion in the dipole [1],

$$
\varepsilon_{x}^{*}=\frac{C_{q} \gamma_{L}^{2} \theta^{3}}{12 \sqrt{15} J_{x}}
$$

with the condition

$$
\begin{aligned}
& \beta_{0}^{*}=\frac{L_{B}}{2 \sqrt{15}}, \quad \alpha_{0}^{*}=0, \quad D_{0}^{*}=\frac{L_{B}^{2}}{24 \rho}, \\
& D_{0}^{\prime *}=0, \quad \mu_{x}^{*}=142 \text { degrees },
\end{aligned}
$$

where quantities with subscript 0 indicate the optical functions at the dipole center, $\mu_{x}$ is the phase advance per half cell, and an asterisked quantity means the quantity is evaluated when the TME condition is fulfilled.

In this paper, TME lattice refers to a lattice beam line consisting of multiple standard cells (cell number $\geq 2$ ) and two matching cells to the dispersion-free straight section at the two ends of the beam line. Each standard cell contains one uniform or combined-function dipole, with the optical functions satisfying condition (4). The bending angle of the dipole in the matching cell is set to $3^{-1 / 3}$ of that in the standard cell to preserve the minimal emittance [3,7]. Other than this type of lattice, double bend achromat (DBA) and triple bend achromat (TBA) lattices are usually adopted in the low-emittance synchrotron radiation storage ring design. The latter types of lattices allow dispersionfree straight sections after every two or three dipoles, however, with minimum emittance of 2 to 3 times of the 
theoretical minimum [8]. In this paper, we address a modified-TME lattice, and will not address the DBA and TBA lattices.

From Eq. (3), the TME emittance is proportional to $\theta^{3}$. To reduce the horizontal natural emittance of an electron storage ring composed of TME cells and with fixed beam energy and circumference, the most effective way is to reduce the bending angle of the dipole, which means increasing the number of the dipoles $N_{d}$ as well as the number of the cells $N_{c}=N_{d}$ in a ring associated with decreasing cell length. (Another effective way is to install damping wigglers in the storage ring [9], which, however, will not be discussed in this paper.) Assume the periodic cells are identical throughout the ring, the total natural chromaticities and average dispersion scale with $N_{d}$ as $\xi_{x, y} \propto N_{d}$ and $\langle D\rangle \propto N_{d}{ }^{-1}$ [10], therefore the chromaticity-correction sextupole's strength $S \propto \xi / D(s)$ is approximately proportional to $N_{d}^{2}$, where $D(s)$ is the dispersion at position of the sextupoles. Summarize the relationship as follows:

$\varepsilon_{x} \propto \theta^{3} \propto N_{d}^{-3} \propto N_{c}^{-3}, \quad \xi \propto N_{c}, \quad\langle D\rangle \propto N_{c}^{-1}, \quad S \propto N_{c}^{2}$.

One can obtain a rough scaling relationship $S \propto \varepsilon_{x}{ }^{-2 / 3}$, which shows that decreasing the emittance in an electron storage ring with TME lattice unavoidably leads to an increase of the required sextupole strengths. In the recent efforts of pushing down the emittance to several tens of picometers region [7,11,12], the required sextupole strengths will be so strong that the nonlinear dynamics of the ring associated with the very large nonlinear geometric and chromatic aberrations from the sextupoles becomes a great challenge for the performance of the storage ring. The dynamic aperture might then be limited to a few millimeters or even smaller, which implies low injecting efficiency and short beam lifetime. It is essential to control the natural chromaticities and the corresponding sextupole strengths in an electron storage ring lattice with extreme-low emittance to facilitate the further nonlinear optimization. Thus, TME-like lattices, namely, lattices with the same or similar configurations as TME lattice and design parameters close to, rather than at, the exact TME condition [Eq. (4)], are usually adopted in a compromised practical design to reach a balance between the extreme-low emittance and moderate natural chromaticities.

Four parameters are usually used to describe the efficiency of a TME-like lattice, namely, the phase advance per half cell $\mu_{x}$, relative emittance $\varepsilon_{r}$, relative beta function $\beta_{r}$, and relative dispersion $D_{r}$,

$$
\varepsilon_{r}=\frac{\varepsilon_{x}}{\varepsilon_{x}^{*}}, \quad \beta_{r}=\frac{\beta_{0}}{\beta_{0}^{*}}, \quad D_{r}=\frac{D_{0}}{D_{0}^{*}} .
$$

The expressions of $\varepsilon_{r}$ and $\mu_{x}$ in terms of $D_{r}$ and $\beta_{r}$ are given by [13-15]

$$
\begin{gathered}
\varepsilon_{r}=\frac{9-10 D_{r}+5 D_{r}^{2}+4 \beta_{r}^{2}}{8 \beta_{r}}, \\
\tan \mu_{x}=\frac{2 \sqrt{3}}{\sqrt{5}} \frac{\beta_{r}}{D_{r}-3} .
\end{gathered}
$$

Yet, one cannot examine the variations of $\varepsilon_{r}$ with respect to $\mu_{x}$ unless we obtain the relationship between $\beta_{r}$ and $D_{r}$.

Figure 1 shows the layout of a typical TME-like cell which consists of one uniform or combined-function dipole and symmetric quadrupole structure outside the dipole, with horizontally focusing quadrupole $(\mathrm{QF})$ closer to the dipole. The horizontal phase advance per half cell is usually varied between $\pi / 2$ and $\mu^{*}=142$ degrees by tuning the quadrupole strengths and drift lengths. The optics in such a TME-like cell has been extensively investigated in previous studies (e.g., $[13,14])$. Because there is no definite relationship between $\beta_{r}$ and $D_{r}$, an additional correlation, $d D_{r} / d \beta_{r}=0$, was introduced to derive concrete relationship between $\varepsilon_{r}$ and $\mu_{x}$ with maximized $D_{r}$ for a given value of $\varepsilon_{r}$.

In this paper, we concentrate on another type of TMElike cell, i.e., with horizontally defocusing quadrupole (QD) closer to the dipole, as shown in Fig. 2. The limitation of the available phase advance of such type of TME-like cell is approximately $\pi / 2$ per half cell [14]. In order to distinguish these two types of TME-like cells, we call the TME-like cell with QF closer to the dipole a conventional-TME cell and that with QD closer to the dipole a modified-TME cell.

We demonstrate that a modified-TME cell promises more compact layout than a conventional-TME cell in the Appendix; and in Sec. II, we show that the relationship between $\beta_{r}$ and $D_{r}$ is not arbitrary but follows an empirical scaling law for a modified-TME cell with uniform dipole,

$$
\frac{\beta_{r}^{2}}{D_{r}-3} \approx \frac{5}{2}+\frac{15}{2 L_{B}[m]} \text {. }
$$

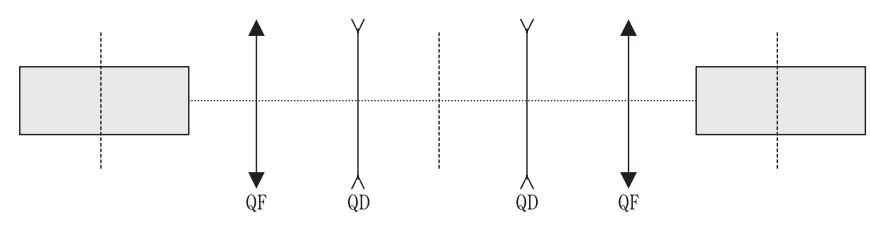

FIG. 1. Layout of a typical TME-like cell, with QF closer to the dipole.

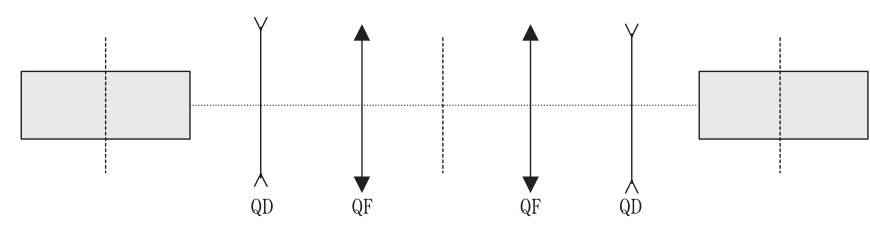

FIG. 2. Layout of another TME-like cell, with QD closer to the dipole. 
With this scaling law and Eqs. (7) and (8), one can examine the parameter variation behaviors and find the optimal $\mu_{x}$ that corresponds to a local minimal $\varepsilon_{r}$ which is of the order of about 3 times of the TME. In addition, due to the found scaling law, the natural chromaticities of such a modifiedTME cell can be exactly solved with a simplified model. The properties of such a lattice configuration are summarized and compared with that of a TME cell, and it shows that this type of lattice appears a good candidate for storage ring-based light source with extreme-low emittance. Then we show in Sec. III that the proposed lattice configuration can be further compressed by combining the horizontally defocusing gradient into the dipole, which also helps reduce the natural chromaticity and the emittance. An empirical scaling law for the relationship between $\beta_{r}$ and $D_{r}$ for a modified-TME cell with combined-function defocusing dipole is also presented. Finally, applications of such a type of lattice in PEP-X light source storage design are presented as demonstration.

\section{MODIFIED-TME CELL WITH UNIFORM DIPOLE}

The dipole magnets in a storage ring bend the charged particles' orbit and therefore define the layout of the ring. The dipole length and bending angle are usually determined at the beginning of concrete optics design effort.

Here we model a modified-TME cell as (Ptd, HD, HD, Ptd), where HD represents half of the dipole, and Ptd represents the part outside the dipole, including drifts and quadrupoles.

The 3-by-3 transfer matrices of the horizontal motion which include the propagation of off-momentum rays for the HD (in small-angle approximation) [16] and Ptd are

$$
\begin{gathered}
M_{\mathrm{hd}}=\left(\begin{array}{ccc}
1 & L_{B} / 2 & L_{B}^{2} / 8 \rho \\
0 & 1 & L_{B} / 2 \rho \\
0 & 0 & 1
\end{array}\right), \\
M_{\mathrm{ptd}}=\left(\begin{array}{ccc}
m_{11} & m_{12} & 0 \\
m_{21} & m_{22} & 0 \\
0 & 0 & 1
\end{array}\right) .
\end{gathered}
$$

Without loss of generality, $m_{11}, m_{12}$, and $m_{21}$ are set to be arbitrary values, while $m_{22}$ is determined by the condition Det $M_{\text {ptd }}=1$ [6].

Here and in what follows, we will constrain the derivation to the case of a compact modified-TME cell with symmetric quadrupole structure and $\mu_{x}$ below $\pi / 2$. The case of $\mu_{x}$ above $\pi / 2$ for a conventional-TME cell has been analyzed in Ref. [17] and elsewhere [14], but will not be specifically discussed in this paper.

Calculate the 2-by-2 transfer matrix of the half cell $M_{\mathrm{hc}}=M_{\mathrm{ptd}} \cdot M_{\mathrm{hd}}$ and write it in terms of Courant-Snyder parameters,

$$
\begin{aligned}
& \left(\begin{array}{lc}
m_{11} & \frac{L_{B}}{2} m_{11}+m_{12} \\
m_{21} & \frac{L_{B}}{2} m_{21}+m_{22}
\end{array}\right) \\
& \quad=\left(\begin{array}{cc}
\sqrt{\frac{\beta_{c b}}{\beta_{0}}} \cos \mu_{x} & \sqrt{\beta_{c b} \beta_{0}} \sin \mu_{x} \\
-\sin \mu_{x} / \sqrt{\beta_{0} \beta_{c b}} & \sqrt{\frac{\beta_{0}}{\beta_{c b}}} \cos \mu_{x}
\end{array}\right),
\end{aligned}
$$

where " $c b$ " indicates cell boundary. The condition of $\alpha=0$ at the dipole center is used to obtain the matrix on the right-hand side of Eq. (12).

From the one-to-one correspondence of the matrix components in Eq. (12), we obtain

$$
\beta_{0} \tan \mu_{x}=\frac{L_{B}}{2}+a,
$$

with $a=m_{12} / m_{11}$, whose unit is meter.

Although the value of $a$ varies with concrete lattice designs, we can still find its variable range. Since $\beta_{0}$ is always positive, the left-hand side of Eq. (13) is positive for $\mu_{x}<\pi / 2$, the minimum $a$ is therefore $-L_{B} / 2$. On the other hand, $\beta_{c b}$ can be obtained by calculating the evolution of the vector $(\beta, \alpha, \gamma)^{T}$ consisting of Courant-Snyder parameters from the dipole center to the cell boundary [18]; the result is

$$
\beta_{c b}=m_{11}^{2}\left[\frac{\beta_{r} L_{B}}{2 \sqrt{15}}+\frac{2 \sqrt{15}}{\beta_{r} L_{B}}\left(a+\frac{L_{B}}{2}\right)^{2}\right] .
$$

For nonzero $m_{11}$ (see below), $\beta_{c b}$ will increase with $a$. The maximum beta function (and of course $\beta_{c b}$ ) is usually set to a moderate value (typically less than $35 \mathrm{~m}$ ) to ensure low sensitivity to vibrations and errors, which imposes a practical limitation to the maximum $a$.

When $\mu_{x}$ approaches $\pi / 2$, through Eqs. (8) and (13), we know that $D_{r}$ tends to 3 , and either $m_{11}$ or $\beta_{0}$ tends to 0 . However, from Eq. (14), if $m_{11}$ tends to $0, \beta_{c b}$ will also tend to 0 , the optics of the modified-TME cell will be unstable. Furthermore, in a practical design of a compact modified-TME lattice (see Fig. 2), the drifts that accommodate sextupoles and other necessary devices, e.g., beam position monitors and vacuum pumps, are usually set to available minimal lengths with small variable range; the quadrupoles QD and QF are used to squeeze the beta and dispersion functions at the dipole center, control the maximal beta functions, and optimize the phase advance to minimize the nonlinear effects. There are usually not enough variables to realize the condition $m_{11}=0$ while satisfying the goals listed above. On the other hand, the other condition, $\beta_{0} \rightarrow 0$, predicts a decreasing of $\beta_{0}$ as $\mu_{x}$ of a compact modified-TME cell increases towards $\pi / 2$.

Combine Eqs. (4), (6), (8), and (13) to eliminate $\mu_{x}$,

$$
\frac{\beta_{r}^{2}}{D_{r}-3}=\frac{5}{2}+\frac{5 a[m]}{L_{B}[m]},
$$

and then substitute the above equation into Eq. (7) to eliminate $D_{r}$; we obtain 


$$
\varepsilon_{r}=\frac{1}{2}\left(\beta_{r}+\frac{1}{\beta_{r}}\right)+\frac{\beta_{r}}{10}\left(\frac{L_{B}[m] \beta_{r}}{2 a[m]+L_{B}[m]}+\frac{5}{\beta_{r}}\right)^{2}
$$

One can see that no matter what the value of $a$ is, the relative emittance $\varepsilon_{r}$ will be large if $\beta_{r}$ is much smaller or larger than 1. If $a$ is smaller than zero, or even close to $-L_{B} / 2, \varepsilon_{r}$ will also be large. Therefore only moderate $\beta_{r}$ and positive $a$ allow the interesting cases for small $\varepsilon_{r}$.

Table I and Fig. 3 show the variation of $\varepsilon_{r}$ with $\beta_{r}$ while setting $L_{B}=2 \mathrm{~m}$ and varying $a$ from 1 to $50 \mathrm{~m}$. As can be seen, the relationship between $\varepsilon_{r}$ and $\beta_{r}$ depends little on $a$, especially for small $\beta_{r}$. It is therefore possible to find an approximate scaling law for the relationship between $\varepsilon_{r}$ and $\beta_{r}$.

We then undertake numerical simulations to verify the analysis. With the modified-TME cell shown in Fig. 4, we randomly vary the normalized quadrupole gradients in a range of $4 \mathrm{~m}^{-2}$ around the original values and drift lengths in a range of $1 \mathrm{~m}$ (but keep the total cell length not greater than $7.5 \mathrm{~m}$ ), record all the stable solutions with $\varepsilon_{r}$ smaller than 10, and plot them in Fig. 5. One can see clearly a linear relationship between $\beta_{r}^{2}$ and $\left(D_{r}-3\right)$. The slope $A$ is obtained by fitting the data.

After simulations of different cases with dipole length from 0.8 to $5 \mathrm{~m}$, as shown in Fig. 6, we find that Eq. (11) with $a=7.73 / 5 \approx 1.5 \mathrm{~m}$ can well describe the variation behaviors of $\beta_{r}^{2}$ with $\left(D_{r}-3\right)$ in a modified-TME cell.

With straightforward derivation, we obtain an approximate expression of $\varepsilon_{r}$ as a function of $\mu_{x}$ and $L_{B}$,

$\varepsilon_{r} \cong \frac{\sqrt{15}}{\tan \mu_{x}}+\frac{\sqrt{3} \tan \mu_{x}}{\sqrt{5} B}+\frac{\sqrt{15}}{2}\left(\frac{1}{\tan \mu_{x}}+\frac{3}{\tan ^{3} \mu_{x}}\right) B$,

with $B=1+3 L_{B}[m]$.

Behaviors of $\beta_{r}$ and $D_{r}$ as functions of $\mu_{x}$ and $L_{B}$ can also be derived. They are omitted here due to their lengthy expressions.

Once the dipole length is determined, one can evaluate and examine the variation behaviors of $\varepsilon_{r}, \mu_{x}, D_{r}$, and $\beta_{r}$ regardless of the concrete settings of the beam line elements outside the dipole. This is very useful for choosing an optimal phase advance of a modified-TME cell. Figure 7 presents the parameter scaling curves for modified-TME lattice with different dipole lengths and $\mu_{x}$ from 60 to 90 degrees. There is a local minimal $\varepsilon_{r}(3-4)$ at $\mu_{x}$ around

TABLE I. $\quad \varepsilon_{r}$ versus $\beta_{r}$, with $L_{B}=2 m$ and $a$ varying from 1 to $50 \mathrm{~m}$.

\begin{tabular}{lcccc}
\hline \hline$\beta_{r}$ & $\varepsilon_{r}(\max )$ & $\varepsilon_{r}(\min )$ & $\varepsilon_{r}($ average $)$ & $\Delta \varepsilon_{r}$ \\
\hline 0.5 & 6.50 & 6.27 & 6.38 & 0.12 \\
1 & 4.02 & 3.53 & 3.77 & 0.25 \\
2 & 4.68 & 2.56 & 3.61 & 1.05 \\
3 & 6.35 & 2.83 & 4.59 & 1.75 \\
4 & 6.35 & 2.83 & 4.59 & 1.76 \\
5 & 8.73 & 3.20 & 5.96 & 2.76 \\
\hline \hline
\end{tabular}

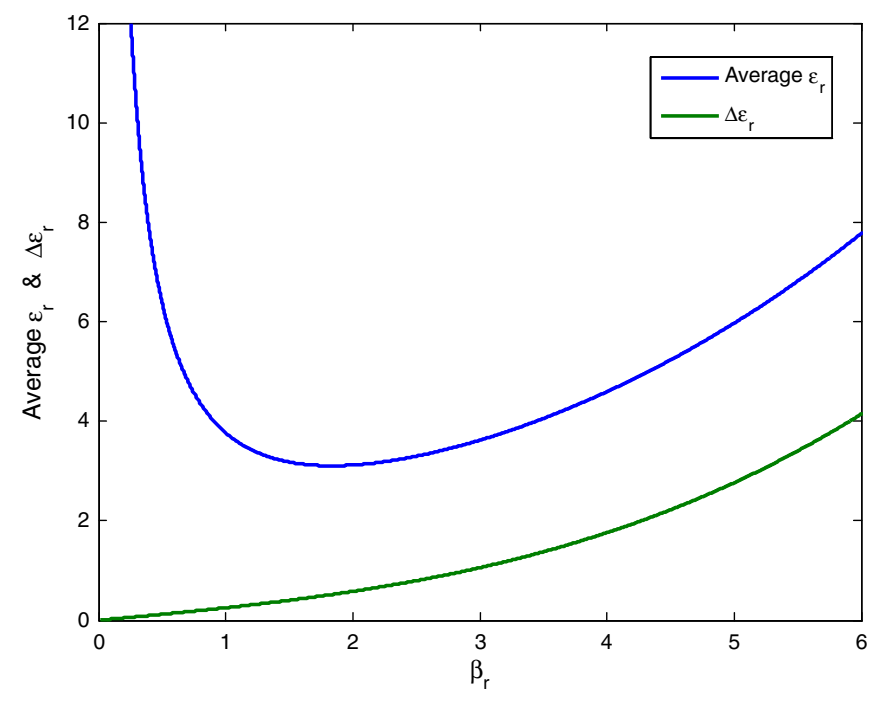

FIG. 3. Average $\varepsilon_{r}$ and $\Delta \varepsilon_{r}$ versus $\beta_{r}$ with $L_{B}=2 \mathrm{~m}$ and $a$ varying from 1 to $50 \mathrm{~m}$.

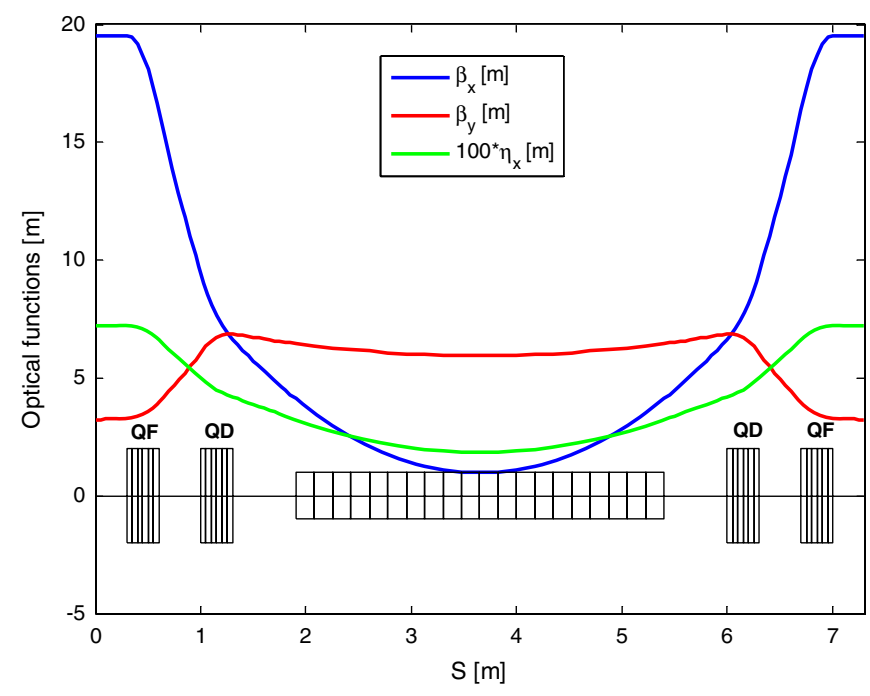

FIG. 4. Optical functions in one modified-TME cell. The cell length is $7.3 \mathrm{~m}, L_{B}=3.5 \mathrm{~m}, \theta=\pi / 99, D_{r}=4, \beta_{r}=2.16$, $\varepsilon_{r}=3.9$, and $\mu_{x}=73.4$ degrees.

80 degrees. It also shows that the shorter the dipole is, the lower the local minimal $\varepsilon_{r}$, and slightly larger the $D_{r}$ and $\beta_{r}$ (for same phase advance) will be. However, according to Eqs. (4) and (6), $\beta_{0}$ and especially $D_{0}$ will decrease quickly with the decreasing dipole length, which in turn requires strong focusing and makes it difficult for a practical lattice design. In addition, the longer dipole allows a greater momentum compaction factor for a longer bunch. Therefore attention should be paid in looking for an appropriate dipole length.

One important issue is the natural chromaticities which are closely related to both linear optics and nonlinear optimization. Unfortunately, to the best of knowledge of the authors, there is not a simple analytic expression for the 


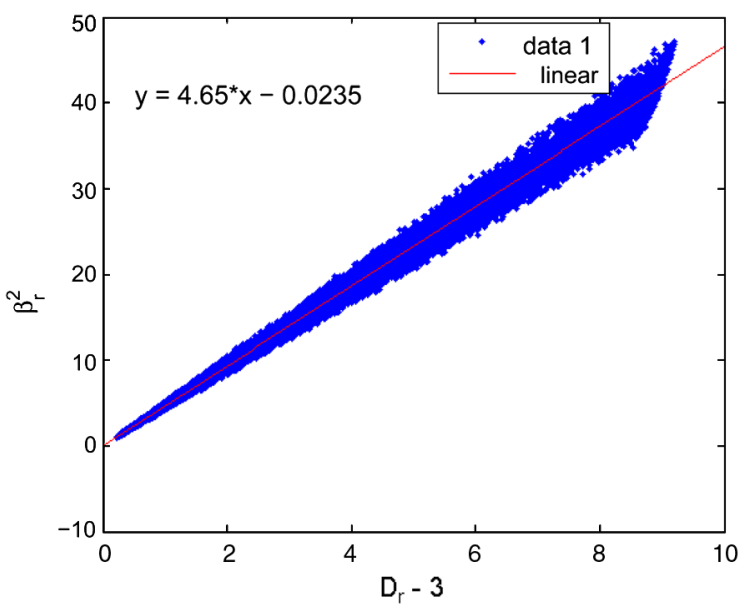

FIG. 5. $\beta_{r}^{2}$ versus $\left(D_{r}-3\right)$ of the simulation results with $\varepsilon_{r}<10$ and $L_{B}=3.5 \mathrm{~m}$.

chromaticity in a TME-like cell, due to more variables and constraints than that in a typical unit cell which consists of a QF and a QD separated by drifts or dipoles [19]. However, for a modified-TME cell with QD closer to the dipole, thanks to the found empirical scaling law for the relationship between $\beta_{r}$ and $D_{r}$, the chromaticities can be obtained using a simplified model. In the model, the quadrupoles are assumed to have vanishing length; space between two QDs is filled by homogeneous-field dipole; maximum horizontal $\beta$ is set to $20 \mathrm{~m}$ and vertical phase advance is matched to be $1 / 3$ of the horizontal. For given values of $L_{B}$ and $\beta_{r}$ (or $D_{r}$ ), one can calculate the $D_{r}$ (or $\left.\beta_{r}\right), \mu_{x}$, and $\varepsilon_{r}$ with the design parameter scaling formulas, then determine the quadrupole strengths and drift lengths using the intrinsic constraints on optical functions, i.e. $\alpha_{0}=0, D^{\prime}{ }_{0}=0, \alpha_{c b}=0, D^{\prime}{ }_{c b}=0$, and constraints on maximum horizontal $\beta$ and vertical phase advance

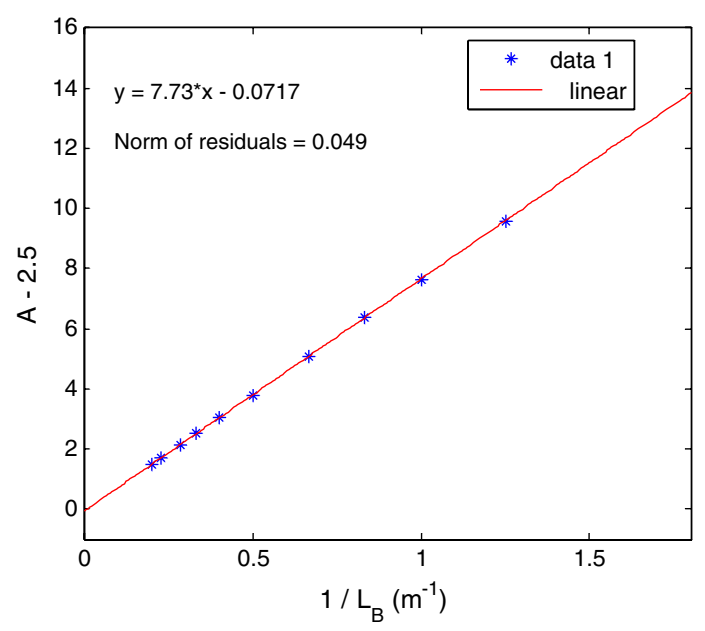

FIG. 6. $(A-2.5)$ versus $1 / L_{B}, A$ is the linear slope between the data of $\beta_{r}^{2}$ and $\left(D_{r}-3\right)$ of the simulations with different dipole length.

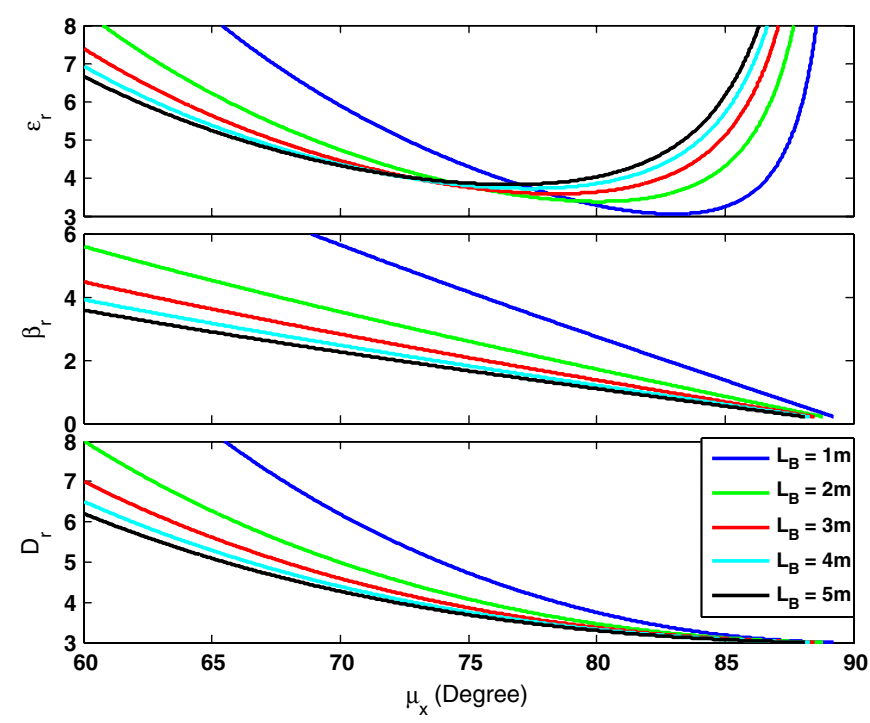

FIG. 7. Parameter scaling curves in modified-TME lattice with different dipole lengths.

mentioned above, and finally obtain the natural chromaticities.

Figure 8 illustrates the dependence of natural chromaticities on $\mu_{x}$ for a modified-TME cell with dipole length $L_{B}=2 \mathrm{~m}$ and $4 \mathrm{~m}$. The variations of $\varepsilon_{r}$ with $\mu_{x}$ are also plotted. For the optimal $\mu_{x}$ corresponding to the local minimum $\varepsilon_{r}$, the horizontal chromaticity is between -1.5 and -1.0 and vertical is below -0.5 . In addition, longer dipoles lead to smaller horizontal chromaticity. In contrast, either horizontal or vertical chromaticity for a conventional-TME cell at the exact TME condition is typically higher than -2 .

In overview, to design an extreme-low-emittance storage ring using modified-TME cells with QD closer to the dipole, the achievable minimum $\varepsilon_{r}$ is about 3 , and it requires $40 \%$ more cells than using TME cells to reach

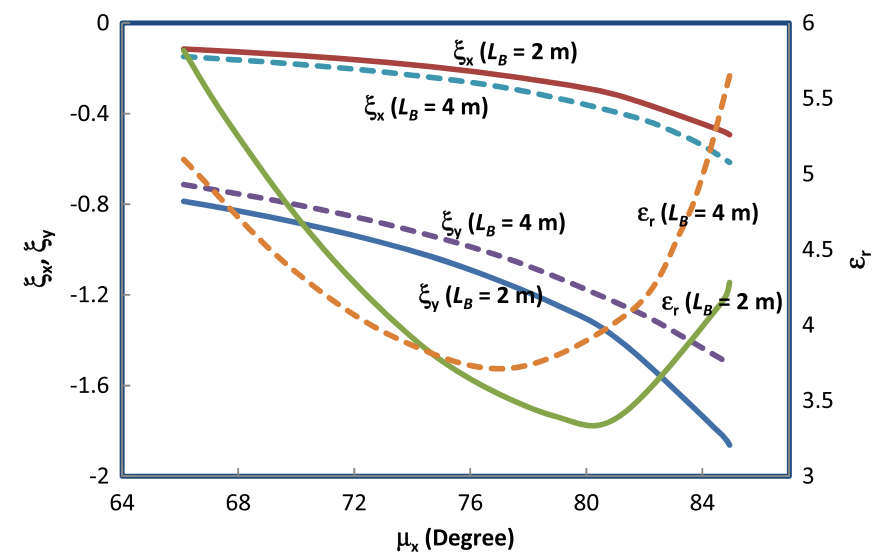

FIG. 8. Horizontal and vertical chromaticities and relative emittance $\varepsilon_{r}$ versus the phase advance per half cell $\mu_{x}$ for $L_{B}=2 \mathrm{~m}$ (solid lines) and $4 \mathrm{~m}$ (dashed lines). 
the same emittance. However, the circumference will be well controlled due to more compact configuration of modified-TME cell than TME cell, and the dynamic aperture optimization will also benefit much from the lower chromaticities and larger dispersion $\left(D_{r}>3\right)$ per cell.

\section{MODIFIED-TME CELL WITH COMBINED- FUNCTION DEFOCUSING DIPOLE}

The proposed configuration, i.e., modified-TME cell with QD closer to the dipole, can be simplified by combining the horizontally defocusing gradient $K$ into the dipole. There are some advantages by using such a configuration. First, the cell length can be further compressed. Second, it is empirically found that the defocusing gradient in the dipole helps reduce the natural chromaticity [14]. Finally, the horizontal damping partition number can be greater than 1 (longitudinal damping partition number will be smaller than 2 at the same time) and increases with the increasing defocusing gradient, leading to lower theoretical minimal emittance than that in a modified-TME cell with normal dipole [17]. For instance, when $K^{1 / 2} L_{B}=1$, the theoretical minimal emittance is $89 \%$ of that using the uniform dipole.

With a similar procedure to that in Sec. II, we obtain the empirical scaling law for the relationship between $\beta_{r}$ and $D_{r}$ that only depends on the dipole length and the defocusing gradient,

$$
\begin{aligned}
& \frac{\beta_{r}^{2}}{\left(D_{r}-\frac{t-C t)}{t-S}\right)} \\
& \cong \frac{(t-S)(t-S C)\left(3 C t+L_{B}[m] S\right)}{S\left(t^{2}+S C t+1-S^{2}-C^{2}\right)\left(3 S t+L_{B}[m] C\right)},
\end{aligned}
$$

where $t=K^{1 / 2} L_{B} / 2, S=\sinh (t)$, and $C=\cosh (t)$. On the limit $t \rightarrow 0$, the above equation tends to Eq. (9). The variation behaviors of the design parameters for a modified-TME cell with combined-function defocusing dipole can be examined in a similar way (for more detail, see [17]).

\section{APPLICATIONS OF MODIFIED-TME LATTICE IN PEP-X STORAGE RING DESIGN}

A future $4.5 \mathrm{GeV}$ light source PEP-X was proposed to be built in the PEP-II tunnel, which has $1.5 \mathrm{~km}$ arclength.

We explore the available minimum emittance of the PEP-X storage ring. With rough estimation, the TME is about 3 pm with $\theta=\pi / 180$. Therefore 390 combinedfunction defocusing dipoles (including 60 half angle dipoles) are used. We divide the arc into 30 periods with straight section of $5 \mathrm{~m}$, and adopt 11 standard modifiedTME cells and two matching cells in each period. On average, each cell is $3.5 \mathrm{~m}$ long, which requires a compact

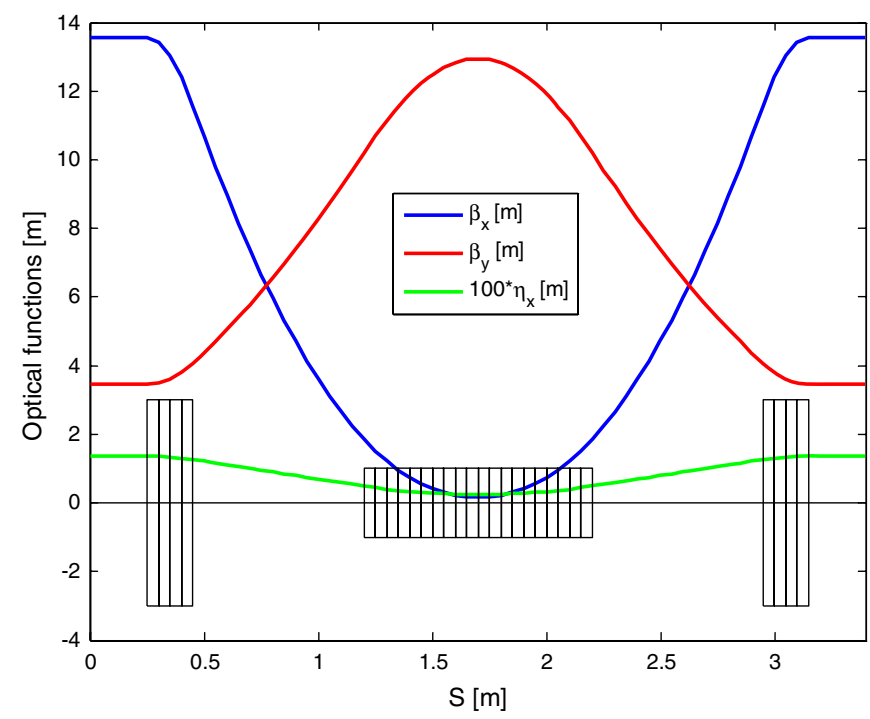

FIG. 9. Optical functions in one standard modified-TME cell for PEP-X storage ring.

layout. Figure 9 shows the optics of one standard $3.4 \mathrm{~m}$ modified-TME cell. Combined-function dipoles with length of $1.1 \mathrm{~m}$ are adopted. The length of QF is minimized by using small aperture magnets that are adopted in MAX IV storage ring design [20]. The phase advance per half cell is chosen to be $\left(\mu_{x}, \mu_{y}\right)=(5 / 11,1 / 11) \pi$ to cancel the geometric aberrations induced by the chromaticitycorrection sextupoles. The lattice has emittance of 9.9 pm with $\varepsilon_{r}=3.3$ and natural chromaticities $\left(\xi_{x}, \xi_{y}\right)=$ $(-1.5,-0.4)$ per cell. Well separated beta functions at the exit of the dipole and the cell boundary and somewhat relaxed relative dispersion $\left(D_{r} \approx 3.1\right)$ help reduce the chromaticity-correction sextupole strengths to below $500 \mathrm{~m}^{-3}$, which can be realized by using sextupoles with full apertures of $20 \mathrm{~mm}$. We choose to treat this lattice design as a conceptual design to demonstrate the capability of a modified-TME lattice to produce extreme-low emittance while keeping the chromaticity-correction sextupole strengths at a large but acceptable level, rather than to use it as a practical optimal design. In particular, due to the very short cell length, the complexity and cost of beam line might be relatively high for this design.

Modified-TME cells with uniform dipoles were adopted in the baseline design for PEP-X storage ring $(2 / 3$ of the total arclength) with horizontal natural emittance of $86 \mathrm{pm}$ $[12,21]$. Each standard modified-TME cell has cell length of $7.3 \mathrm{~m}$ and relatively low phase advance, $\left(\mu_{x}, \mu_{y}\right)=$ $(3 / 8,1 / 8) \pi$ per half cell, which provides cancellation of chromatic and sextupole aberrations in every eight cells. The natural chromaticities $\left(\xi_{x}, \xi_{y}\right)$ are $(-0.7,-0.25)$ per cell. The strengths and integral strengths of the sextupoles used in the lattice design are below $70 \mathrm{~m}^{-3}$ and $15 \mathrm{~m}^{-2}$, respectively. The dynamic apertures (considering the magnetic field errors) for particles with momentum deviations 
of $(-3 \%, 3 \%)$ are about $5 \mathrm{~mm}$ in the horizontal plane and $1 \mathrm{~mm}$ in the vertical plane.

\section{CONCLUSION}

To push down the emittance of an electron storage ring to the level of several tens of picometers, it requires TME-like lattice with compact configuration and moderate phase advance to avoid excessive strong sextupoles. In this paper, we demonstrate that the modified-TME cell with defocusing quadrupole closer to the dipole, or simply with combined-function defocusing dipole, allows minimal emittance of the order of about 3 times of the theoretical minimum, compact layout, phase advance below $\pi / 2$, relaxed optical functions, and relatively low natural chromaticities, which in turn are expected to help produce a practical lattice design with extreme-low emittance and chromaticity-correction sextupoles of moderate strengths. We also present the empirical relationships of the design parameters for such types of lattices. The scaling law and the illustration of the dependence of the natural chromaticities on the phase advance per half cell enable us to evaluate and examine the variation behaviors of the parameters before performing the timeconsuming optics matching, which in turn facilitates the practical lattice design.

\section{ACKNOWLEDGMENTS}

One of us (Yi Jiao) thanks Y. Nosochkov and M. Wang in SLAC, USA, and G. Xu in IHEP, China, for helpful discussions and comments. This work is supported by the
Department of Energy under Contract No. DE-AC0276 SF00515.

\section{APPENDIX: COMPARISON OF TWO CONFIGURATIONS}

The difference between the two types of TME-like cells is the layout of the doublet outside the dipole, as shown in Fig. 10. The upper and lower figures correspond to the TME-like cell with QF and QD closer to the dipole (see Figs. 1 and 2), respectively. In order to simplify the comparison, it is assumed that the two doublets have QF and QD with same strengths and same distances $d$ between the two quadrupoles.

In thin-lens approximation, the combined action of the two quadrupoles is equivalent to that of a single lens with focal length $F$ given by [22]

$$
\frac{1}{F}=\frac{1}{f_{F}}+\frac{d}{f_{F} f_{D}}-\frac{1}{f_{D}},
$$

where $f_{F}$ and $f_{D}$ are the focal length of single lens, they are both positive here.

Although the equivalent focal lengths of two doublets are the same, the locations of the principal planes (dashed lines in Fig. 10) are different. The focal point where the rays cross the axis to the right of the doublet approximately corresponds to the dipole center in a TME-like cell. Therefore one can evaluate the distance from the dipole center to the quadrupole closer to the dipole for these two configurations,

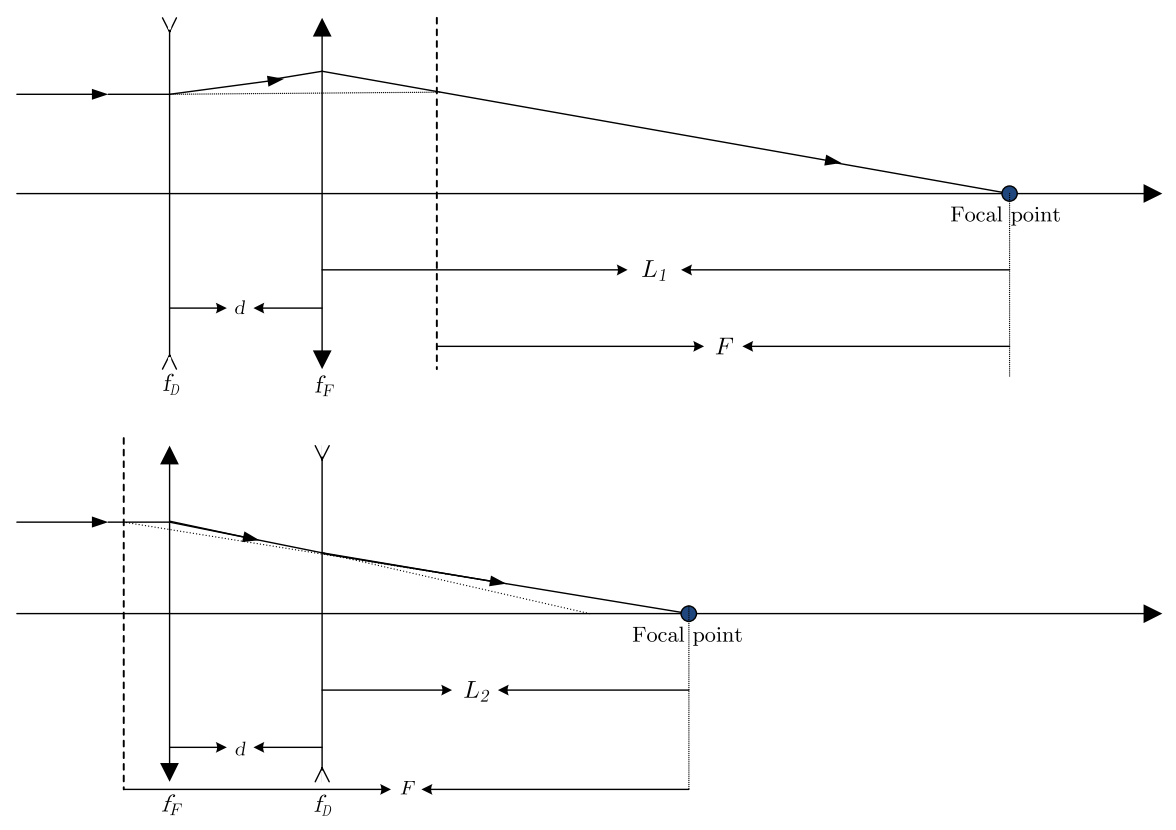

FIG. 10. Sketch view of the doublets, $F$ is the equivalent focal length, $d$ is the distance between the two lenses, $L_{1}$ and $L_{2}$ are the distances between the focal point and the nearest lens. 


$$
L_{1}=F\left(1+\frac{d}{f_{D}}\right) \quad L_{2}=F\left(1-\frac{d}{f_{F}}\right) .
$$

It is clear that $L_{2}<L_{1}$. If the beta and dispersion functions at the dipole center of both configurations can be compressed to the required values, the TME-like cell with QD closer to the dipole is preferred, because it allows more compact layout.

[1] L. C. Teng, Fermilab Report No. TM-1269, 1984.

[2] C.X. Wang, Phys. Rev. ST Accel. Beams 12, 061001 (2009).

[3] S. Y. Lee, Phys. Rev. E 54, 1940 (1996).

[4] D. Einfeld, J. Schaper, and M. Plesko, J. Phys. IV (France) 04, C9-373 (1994).

[5] M. Sands, in Physics with Intersecting Storage Rings, edited by G. Touschek (Academic, New York, 1971), pp. 257-411.

[6] E. D. Courant and H.S. Snyder, Ann. Phys. (Paris) 3, 1 (1958).

[7] K. Tsumaki and N. Kumagai, Nucl. Instrum. Methods Phys. Res., Sect. A 565, 394 (2006).

[8] S. Y. Lee and L. Teng, in Proceedings of the 1991 Particle Accelerator Conference, San Francisco, CA, 1991 (IEEE, New York, 1991), p. 2679.
[9] H. Wiedemann, Nucl. Instrum. Methods Phys. Res., Sect. A 266, 24 (1988).

[10] M. Borland, A Comparison of USRs and ERLs as Hard X-ray Sources, invited accelerator seminar at SLAC, 2011.

[11] M. Borland, Nucl. Instrum. Methods Phys. Res., Sect. A 557, 230 (2006).

[12] Y. Cai et al., Report No. SLAC-PUB-13999, 2010.

[13] J.P. Potier and L. Rivkin, CERN Report No. CERN-PS97-020, 1997.

[14] P. Emma and T. Raubenheimer, Phys. Rev. ST Accel. Beams 4, 021001 (2001).

[15] L. Rivkin, in Proceedings of the 7th International Workshop on Linear Colliders (LC97), Zvenigorod, Russia (INP, Provino, Russia, 1997), p. 644.

[16] A.W. Chao and M. Tigner, Handbook of Accelerator Physics and Enginering (World Scientific, Singapore, 2006), p. 65-66.

[17] Y. Jiao, Y. Cai, and A. W. Chao, Report No. SLAC-PUB14205, 2010.

[18] T.P. Wangler, Principles of RF Linear Accelerators (Wiley, New York, 1998), p. 217.

[19] E. Keil, CERN Report No. 77-13, 1977, p. 11.

[20] S. C. Leemann et al., Phys. Rev. ST Accel. Beams 12, 120701 (2009).

[21] M.H. Wang et al., Nucl. Instrum. Methods Phys. Res., Sect. A (to be published).

[22] M. Reiser, Theory and Design of Charged Particle Beams (Wiley, New York, 1994), p. 115. 